\title{
A scaffold cell seeding method driven by surface acoustic waves
}

\author{
Haiyan Li, James R. Friend*, Leslie Y. Yeo \\ Micro/Nanophysics Research Laboratory, Monash University, Clayton, VIC 3800, Australia
}

Received 7 February 2007; accepted 5 June 2007

\begin{abstract}
Surface acoustic waves (SAW) have been employed to drive a particle suspension into a porous scaffold as a means for cell seeding. Straight, simple interdigital electrode structures were fabricated on lithium niobate to permit the generation of Rayleigh SAW radiation. Fluorescent microscopy was used to investigate the seeding process; the SAW-driven seeding process occurred in approximately $10 \mathrm{~s}$, much quicker than if the scaffold were to be seeded by gravity-driven diffusional processes alone ( $>30$ min). Analysis of high-speed micrographic images demonstrated that the SAW method could also drive particles deeper into the scaffold, thereby significantly improving the uniformity of the particle distribution. The proposed SAW technique therefore offers a promising technology to dramatically improve the speed and uniformity of cell seeding in scaffolds, which might contribute to rapid and uniform tissue regeneration.
\end{abstract}

Crown Copyright (C) 2007 Published by Elsevier Ltd. All rights reserved.

Keywords: Surface acoustic wave; Tissue engineering; Rapid cell seeding; Microfluidic actuation

\section{Introduction}

In order for in vitro cell cultures to take place within the extra-cellular matrices, the desired cells have first to be seeded into the scaffold while at the same time limiting any denaturing or lysing that could potentially render the cells ineffective [1]. Achieving homogeneity in the cultured tissues is also clearly dependent on the uniformity of the cell distribution within the scaffolds and the efficiency of the seeding process. Any significant reduction in the total time required for cell seeding could also lead to substantial savings in cost. Further, an improvement in cell viability may occur since long seeding periods can result in adverse effects in which cells might expire or age beyond their useful state, which, in turn, affects the cells' adhesive, proliferation, and differentiation processes [2].

Given the typical hydrophobicity and small pore sizes (typically $10-150 \mu \mathrm{m}$ ) of polymeric scaffold materials, the imbibitions of a cell suspension into the scaffold in the absence of any external driving forces is exceptionally slow due to the large capillary resistance encountered. Under

\footnotetext{
${ }^{*}$ Corresponding author. Tel./fax: + 61399053551.

E-mail address: James.Friend@eng.monash.edu.au (J.R. Friend).
}

gravitational forces alone, perfusion of a cell suspension into the scaffold typically takes hours to days $[3,4]$. Nevertheless, this static seeding method has conventionally been used in tissue culturing due to its simplicity $[5,6]$. The large capillary resistance also poses considerable difficulty in delivering cells deep within the scaffold structure in the absence of external driving forces. For example, studies have reported that new bone tissue forms easily at the surface of scaffolds but is hard to regenerate in the centre of the scaffolds due to the superficial penetration of the cell suspension with conventional seeding methods resulting in a large proportion of the cells being deposited merely on the surface of the scaffold $[7,8]$.

Several improved methods are currently being explored for seeding cells within scaffolds and achieving a greater homogeneous cell distribution within. These methods typically involve affixing a scaffold in an agitated vessel filled with the cell suspension. The cells then perfuse into scaffold under the velocity gradient arising between the advected cells and the stationary scaffold. However, low seeding efficiencies have been reported in addition to the long seeding times (hours to weeks) to finish a cell seeding process and most of cells aggregated on the surface of the scaffold, which result in a non-uniform cell seeding [9-14]. 
Soletti et al. [1] have proposed a technique for cell seeding in which cells within a suspension flowing through the annulus of a tubular scaffold perfuse through the scaffold under vacuum. The scaffold is concurrently rotated in order to achieve greater distribution uniformity. Although significantly reduced seeding times (several minutes) and improved distribution have been obtained, the use of vacuum and syringe pumps necessarily involves cumbersome equipment.

Recently, surface acoustic wave (SAW) technology has been developed for the use of biotechnology, including biofluidic mixing, particle trapping, and biosensors in microfluidic devices [15-18]. A SAW is like an earthquake wave propagating along the surface with an amplitude of a few tens of nanometers (Fig. 1(a)). The wave is created by an interdigital transducer (IDT) electrode [17] and the wavelength is defined by the width of each individual finger along the propagation direction of the wave and the gap between them. The amplitude of the electrical signal determines the amplitude of the wave. Though there are many forms of SAW, this study makes use of the Rayleigh wave, an axial-surface-normal polarized SAW [19]. There are many examples of the application of SAW technology for sensing [18]; the interaction of a fluid in contact with the propagating radiation allows the measurement of the fluid's viscosity, density and $\mathrm{pH}$ through the appropriate design of the SAW device. Wixforth et al. [20] has

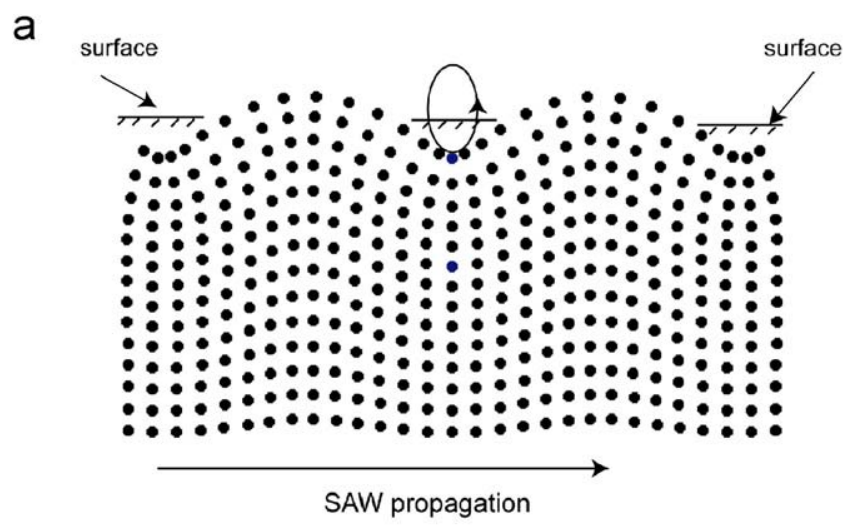

b

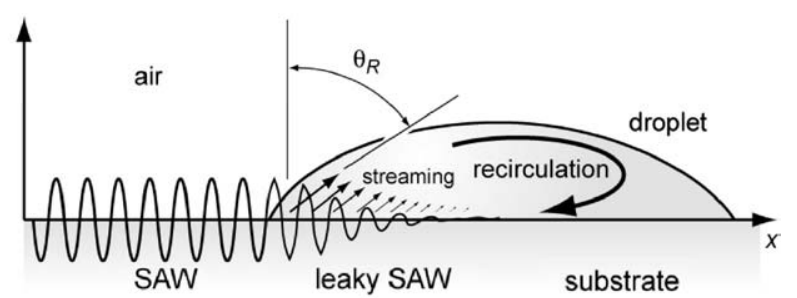

Fig. 1. (a) Sketch illustrating the propagation of SAW through the lithium niobate substrate. The Rayleigh wave is localized to the surface with an exponential decay to a negligible amplitude 4-5 wavelengths below the surface of the substrate. Particle motion on the surface is elliptical in a direction opposite the propagation direction of wave. (b) Acoustic streaming is generated within a small droplet by SAW due to leakage of acoustic energy into the fluid at an angle $\theta_{\mathrm{R}}$ from the Rayleigh wave in the substrate. demonstrated that SAWs strongly interact with small amounts of liquid on the surface of a piezoelectric substrate by inducing bulk liquid recirculation through a process known as acoustic streaming (Fig. 1(b)). SAW entering the droplet is diffracted at the Rayleigh angle $\theta_{\mathrm{R}}$ into the fluid, where it generates a longitudinal pressure wave. For an infinite half-space, this $\theta_{\mathrm{R}}$ is given by the ratio of the sound velocities in the substrate and in the fluid, respectively. If the intensity of the acoustic radiation component into the fluid is sufficiently high, and the liquid consists of a free droplet whose contact line is not pinned, the SAW can induce the droplet to move in the direction of the SAW. The technology is therefore a convenient mechanism for transporting a free droplet on a microfluidic device while requiring no mechanically moving components other than small deformations of the substrate itself. In this work, we demonstrate that SAW actuation can also be exploited as a method for quickly driving particles into a scaffold, providing a means for rapid, uniformly distributed, and efficient cell seeding in tissue engineering applications.

\section{Materials and methods}

\subsection{Scaffolds}

Scaffolds were prepared using a conventional solvent casting-particulate leaching method [4] using polycaprolactone (PCL, MW $=65,000$, $\mathrm{MP}=65^{\circ} \mathrm{C}$ ) (Sigma Chemical Co., USA). Briefly, PCL powders were dissolved in chloroform with a concentration of $10 \%(\mathrm{w} / \mathrm{v})$. Sodium chloride $(\mathrm{NaCl})$ particles sieved as porogens $(100-150 \mu \mathrm{m})$ were then incorporated into the suspension $(\mathrm{NaCl} /$ polymer $=1: 9)(\mathrm{w} / \mathrm{w})$, and the dispersion was cast into a 60 -mm Teflon mould. The samples were airdried under a fume hood for $24 \mathrm{~h}$ to allow the solvent to evaporate and were subsequently vacuum dried at $60^{\circ} \mathrm{C}$ for $48 \mathrm{~h}$ to remove any remaining solvent. Then the samples were immersed in deionized water to leach out porogens in the resulting polymer/salt composites. The samples were finally vacuum-dried to obtain a set of sponge-like scaffolds with thicknesses of $2 \mathrm{~mm}$. Before seeding, the scaffolds were carefully cut with a razor blade into $6 \times 5 \mathrm{~mm}$ squares and were stored in a desiccator. The porosity of the scaffold was about $90 \% \pm 1.5 \%$, determined by using Archimedes' principle described by Yang [21].

\subsection{Fluorescent particles}

An aqueous suspension of $5-\mu \mathrm{m}$ green fluorescent polystyrene (PS) microspheres (Duke Scientific Corporation, USA) was used to facilitate observation of the seeding process and their spatial distribution in the scaffold. The excitation maxima wavelength for these particles is $468 \mathrm{~nm}$ (blue) and the emission maxima wavelength is $508 \mathrm{~nm}$ (green). These PS microparticles were obtained in the form of a $1 \%(\mathrm{w} / \mathrm{v})$ aqueous suspension, containing $1.4 \times 10^{8}$ particles in $1 \mathrm{ml}$. Using deionized (DI) water, the original suspension was diluted to obtain a concentration of $1.4 \times 10^{6}$ particles $/ \mathrm{ml}$.

\subsection{Seeding techniques}

\subsubsection{Particle seeding using $S A W$ radiation}

A method for direct pumping of a fluorescent particle suspension through the pores of $3 \mathrm{D}$ scaffolds using SAW radiation is described. A SAW is excited by the application of a radio frequency (RF) signal to an IDT fabricated onto a piezoelectric substrate. The wavelength of the excited SAW, and therefore its resonance frequency, is defined by the 
geometry of the IDT. A $0.5-\mathrm{mm}$ thick substrate, $127.68^{\circ} \mathrm{YX}$ cut, Xpropagating lithium niobate $\left(\mathrm{LiNbO}_{3}\right.$ or $\mathrm{LN}$, Roditi, London, UK) single crystal was used as the SAW substrate in this experiment to generate a Rayleigh wave upon excitation of the IDT by an RF signal. A 4-nm titanium layer and a 150-nm aluminium layer were sputter-deposited upon this substrate. A standard alternating finger IDT with 60 straight finger pairs and an aperture of $8 \mathrm{~mm}$ was fabricated using photolithography; the strip and gap widths were $49 \mu \mathrm{m}$, thus giving rise to a wavelength of $\lambda=196 \mu \mathrm{m}$. The IDT was driven with a sinusoidal signal at the resonance frequency $f_{0}$, defined as $f_{0}=\mathrm{c} / \lambda$. The SAW velocity $c=3788 \mathrm{~m} / \mathrm{s}$, and hence $f_{0} \approx 19.35 \mathrm{MHz}$. Such an IDT efficiently converts the applied RF signal into an acoustic wave, which in this case travels from the IDT in both directions along the substrate surface and perpendicular to the direction of the strip electrodes with low divergence.

Fig. 2(a) shows a schematic of the experimental setup as seen from above. Situated on the left side is the input IDT. The wires in contact with the bus bars of the IDT are connected to the RF power source. A $20-\mu 1$ drop containing the fluorescent PS suspension with a concentration of $1.4 \times 10^{6}$ particles $/ \mathrm{ml}$ is then deposited onto the surface of the SAW device between the IDT and the scaffold. To observe the fluorescent PS particles, a high-speed video camera (Olympus $i$-speed, Tokyo, Japan) was used through a reflected fluorescent microscope system (Olympus BXFM, Tokyo, Japan) to monitor the dynamics of the seeding process. Wideband blue light with an intensity maximum at $468 \mathrm{~nm}$ was used to excite the particle fluorescence.

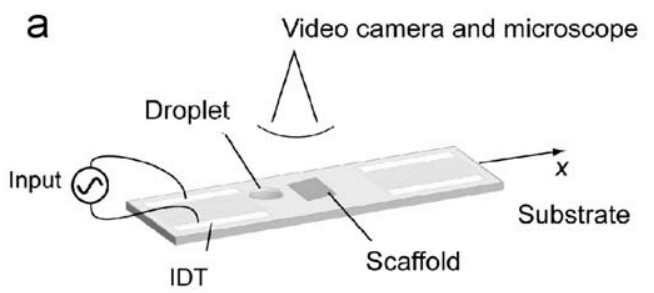

b

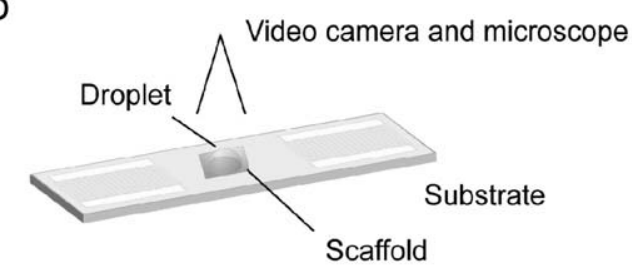

C

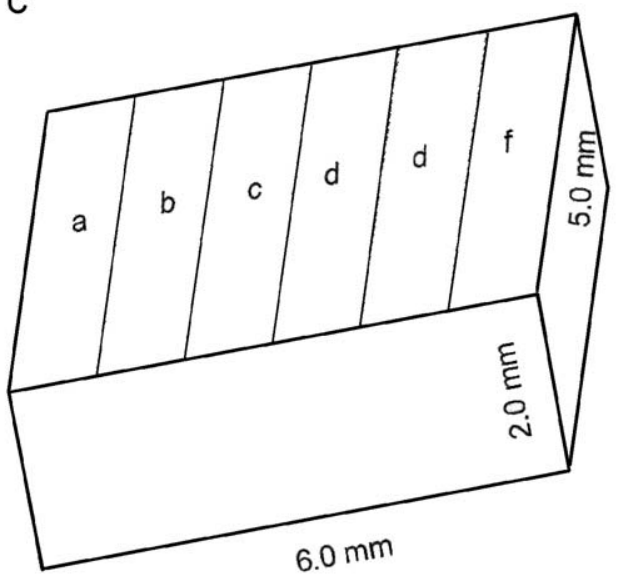

Fig. 2. (a) Schematic of the experimental setup for cell seeding using SAW actuation. (b) Experiment setup for gravity-driven case. (c) Six 1-mm thick sections were cut through the scaffold to evaluate the distribution of the cells within it.

\subsubsection{Static seeding}

In order to benchmark the efficiency of the SAW-driven cell seeding process, we compared the results from Section 2.3.1 above with a static seeding process in which the cell suspension is allowed to perfuse through the scaffold solely by pure diffusion. First, PCL scaffolds were placed onto a SAW substrate. A $20-\mu \mathrm{l}$ drop of fluorescent particulate suspension with a concentration of $1.4 \times 10^{6}$ particles $/ \mathrm{ml}$ was pipetted onto the top surface of the scaffold. The same observation system was used to monitor the seeding process. The approximate time required for the scaffold to completely absorb the droplet suspension, i.e., no liquid apparent above the surface, was determined through visual inspection using the microscope system.

\subsection{Image analysis}

After the seeding process, the scaffolds were cut perpendicularly to the long axis into six 1-mm thick slices (Fig. 2(b)). The cross-section of each slice $(\mathrm{a}-\mathrm{f})$ was then observed under the reflected fluorescent microscope system. Slice (a) is the side where the cell suspension first enters in the two methods described above. The green-colour pixel intensities of each image was acquired using Photoshop CS (Adobe Systems Software Ltd., Ireland); each image's intensity was normalized against the intensity of the image of the initially wetted surface of the scaffold.

\subsection{Particle seeding efficiency}

A digital frame-by-frame analysis of the high-speed video was employed to determine the area of the image under which the fluorescent signal was evident. The velocity was computed by dividing the distance that the drop suspension travelled prior to entry into the scaffold with the time taken. Preliminary investigations were aimed at determining the influence of the input RF power on the suspension's velocity prior to entry into the scaffold and the particle seeding efficiency of the scaffolds. The particle seeding efficiency was evaluated according to the following method: the SAW radiation was terminated when the entire suspension entered the scaffold completely, i.e., no liquid external to the scaffold remained on the SAW surface. None of the liquid was permitted to circumvent the scaffold or to atomize [22]. Generally, the power required to atomize a droplet is higher than that used in this study. The SAW substrate was then rinsed using DI water and the solution was collected. The number of fluorescent particles in this solution, $N_{1}$, was counted by using a hemocytometer (Hausser Scientific, Horsham, PA, Sigma, USA). There were $2.8 \times 10^{4}$ fluorescent particles in the 20 - $\mu$ l droplet according to the original fluorescent particle concentration $\left(1.4 \times 10^{6}\right.$ particles $\left./ \mathrm{ml}\right)$. The "particle seeding efficiency" of the scaffold is then given by

Particle seeding efficiency $\%=100 \times\left(2.8 \times 10^{4}-N_{1}\right) / 2.8 \times 10^{4}$.

\subsection{Statistical analysis}

All values are presented as mean \pm standard deviation. Differences among experimental groups were assessed using a two-tailed Student's $t$ test and considered statistically significant if $p<0.05$.

\section{Results and discussion}

\subsection{Effect of input RF power on the particle seeding efficiency}

Fig. 3 describes the relationship between the velocity at which the particle suspension enters the scaffold and the particle seeding efficiency within the scaffold. Below approximately $325 \mathrm{~mW}$, the particle seeding efficiency is low $(<50 \%)$ as there is insufficient fluid velocity to drive 


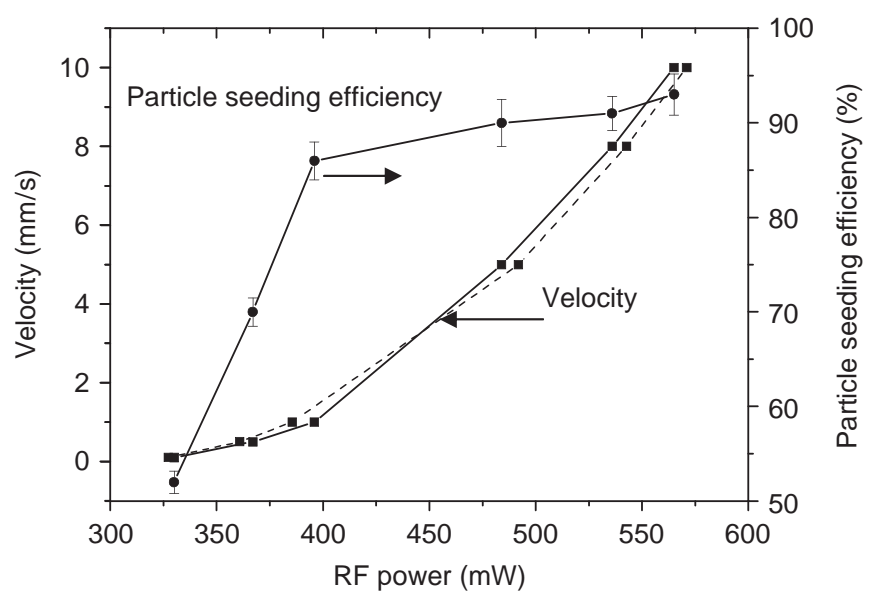

Fig. 3. Effect of input RF power on the velocity of the droplet suspension prior to its contact with and entry into the scaffold, and the particle seeding efficiency within the scaffold. Dash line is the trend line, showing that the relationship between velocity $(u)$ and RF power $(P)$ is $P \sim u^{2}$.

the suspension into the scaffold. The majority of particles were found to aggregate on the SAW substrate. Above this input power, however, there was a marked increase in the particle seeding efficiency, approximately $50-85 \%$ despite only a modest increase in the fluid droplet velocity to $1 \mathrm{~mm} / \mathrm{s}$. Above approximately $400 \mathrm{~mW}$, however, the particle seeding appeared to plateau despite a sharp increase in the droplet velocity as the input RF power was increased. The highest seeding efficiency was about $92 \%$ at an RF power of $570 \mathrm{~mW}$. Above this power, the intense SAW radiation resulted in the atomization of the droplet containing the suspension even before it reached the scaffold. This thus imposes an upper limit on the input RF power. Since there is no significant increase in the seeding efficiency beyond $400 \mathrm{~mW}$, the subsequent experiments were conducted using this input power level.

The increase in the velocity $u$ with the input RF power was observed to be quadratic, which is consistent since the power $P$ scales as

$P \sim u F \sim \rho f R^{3} u^{2}$,

where $F$ is the acoustical force in which $\rho$ denotes the density, $f$ the SAW frequency and $R$ the characteristic drop dimension. The plateau in the cell seeding efficiency above $400 \mathrm{~mW}$, on the other hand, is associated with the elastic-plastic transition for the scaffold, which demonstrates the typical behaviour of porous polymer materials undergoing deformation. For a typical polycaprolactone porous scaffold structure, the yield stress (transition from elastic to plastic behaviour) occurs at about $3 \mathrm{MPa}$, while its stiffness or Young's modulus is about $42 \mathrm{MPa}$ [23]. The energy retained by the scaffold required to make the transition to plastic behaviour, and therefore very lossy characteristics which absorb the SAW radiation is given by

$E=\frac{1}{2} \sigma \varepsilon=\frac{1}{2} \frac{\sigma^{2}}{y}$ where $E$ is the structural energy in the scaffold per unit volume, $\sigma$ is the stress, $\varepsilon$ strain, and $y=42 \mathrm{MPa}$ is Young's modulus. At yield, we therefore obtain $E=1.1 \times 10^{-4} \mathrm{~J} /$ $\mathrm{mm}^{3}$. Compared with the energy put into the system, $E_{\text {in }}=(400 \mathrm{~mW} \times 10 \mathrm{~s}) /\left(6 \times 5 \times 2 \mathrm{~mm}^{3}\right)=0.07 \mathrm{~J} / \mathrm{mm}^{3}$, it is conceivable that this is a possible reason for the plateau, given that some of the energy is lost to electrical resistance, to motion of the fluid droplet, and to opposite end of the substrate beyond the scaffold. In any case, these results indicate that seeding of the scaffolds with a porous architecture can be enhanced through SAW-driven actuation of the particle suspension.

Recent studies have shown that cell's viability will not be damaged when the cells are treated by ultrasonic standing wave fields [24,25]. Studies on freely suspended yeast acoustically treated in ultrasonic standing wave devices for longer periods (up to $2 \mathrm{~h}$ ) suggest that the viability of the yeast cells was not significantly affected [24]. Similarly, no disruption of red blood cells was noted when using a tubular acoustic resonator optimized for diagnostic purposes and subsequently used as an alternative method for concentrating red blood cells [25]. Therefore, there is sufficient evidence to suggest that SAW radiation at comparatively low power levels would not affect the cell viability.

\subsection{Dynamics of seeding process}

High-speed video images of the particle seeding process at $400 \mathrm{~mW}$ have been captured at 60 frames/s. The images in Fig. 4(a) show the geometry of the droplet and scaffold under bright illumination field at $t=0$ prior to seeding. The left image shows the positions of the droplet and scaffold in the SAW-driven method and the right image shows that the droplet was pipetted onto the surface of the scaffold, maintaining a round shape, at least initially. Images in Fig. 4(b), in which the seeding process is observed dynamically under fluoroscopic illumination, show that the SAW-driven particle seeding process is rapid. The green fluorescence indicates that approximately $85 \%$ of the particles in the initial suspension were distributed within the scaffold in just $10 \mathrm{~s}$. We observed the droplet to approach the scaffold and subsequently to be driven into the scaffold by SAW actuation. The fluorescence almost completely disappears in the last image at $t=10 \mathrm{~s}$, indicating that the majority of the fluorescent PS particles have completely entered the scaffold structure.

In contrast, the static seeding process, in which the particle suspension is allowed to perfuse into the scaffold by pure diffusion, requires approximately $30 \mathrm{~min}$ (Fig. 4(c)). Due to the small scaffold pores, roughly $200 \mu \mathrm{m}$, the capillary stress $=\gamma / R_{\mathrm{p}}$, where $\gamma$ is the surface tension and $R_{\mathrm{p}}$ is the pore dimension, is extremely large, on the order of $10^{2} \mathrm{~N} / \mathrm{m}^{2}$. As such, gravity is insufficient to drive the suspension through the scaffold and the suspension permeates the scaffold by pure diffusion alone. 


\section{a}

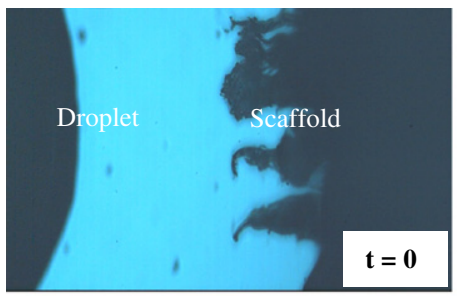

b
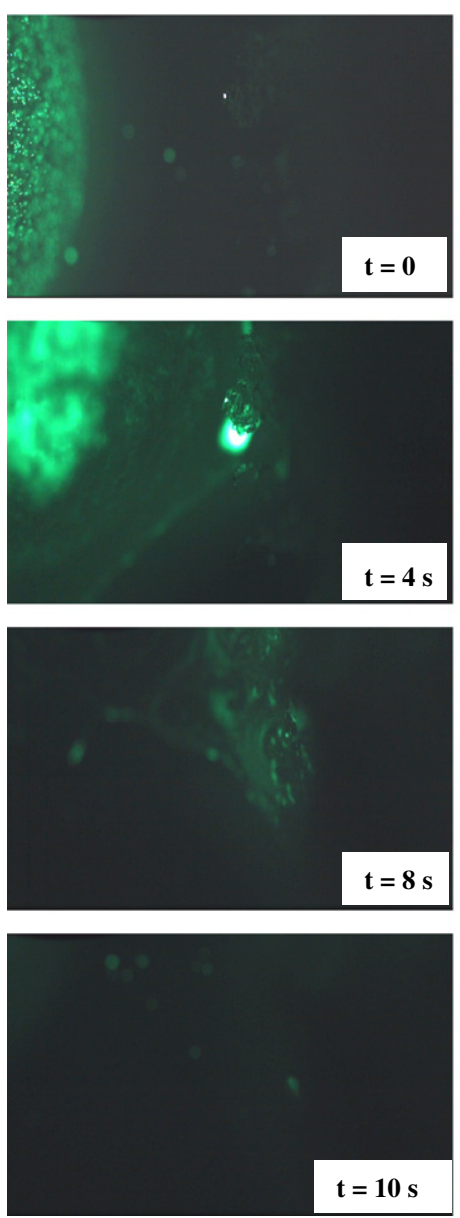

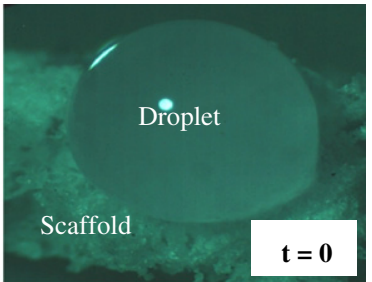

C
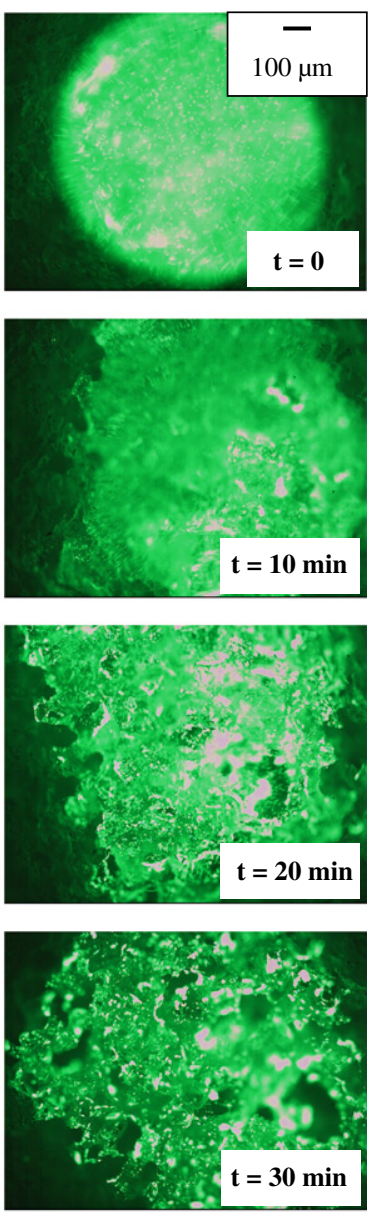

Fig. 4. (a) Geometry of scaffold and droplet under bright illumination. (b) Successive images acquired at 60 frames/s show a fluoroscopic side view of the SAW-driven perfusion process under in roughly $10 \mathrm{~s}$. (c) A plane view of the static method in which the drop perfuses through the scaffold by pure diffusion alone, taking approximately $30 \mathrm{~min}$. All images are at the same magnification.

Fig. 5. (a) Scaffold cross-sectional slices at various positions given in Fig. 2(b). Slice (a) is therefore the slice adjacent to the side where the suspension enters the scaffold. Green fluorescence indicates the presence of particles. The left columns show the SAW-driven scaffold slices. Significant penetration to the last slice was achieved. The right columns are images of the static-loaded slices, showing little particle penetration beyond the first two slices. All images are at the same magnification. (b) Particle distribution in each slice showing significantly greater uniformity in the seeding processes driven by SAW actuation. In both cases, the scaffold dissection was carried out immediately after the entire suspension entered the scaffold, $10 \mathrm{~s}$ for the SAW-driven samples, and $30 \mathrm{~min}$ for the static, gravity-driven samples.

\subsection{Spatial distribution of seeded particles}

The particle seeding efficiencies only provide information about the number of particles that enter the scaffold. By slicing the scaffold into several 1-mm sections, as shown

a
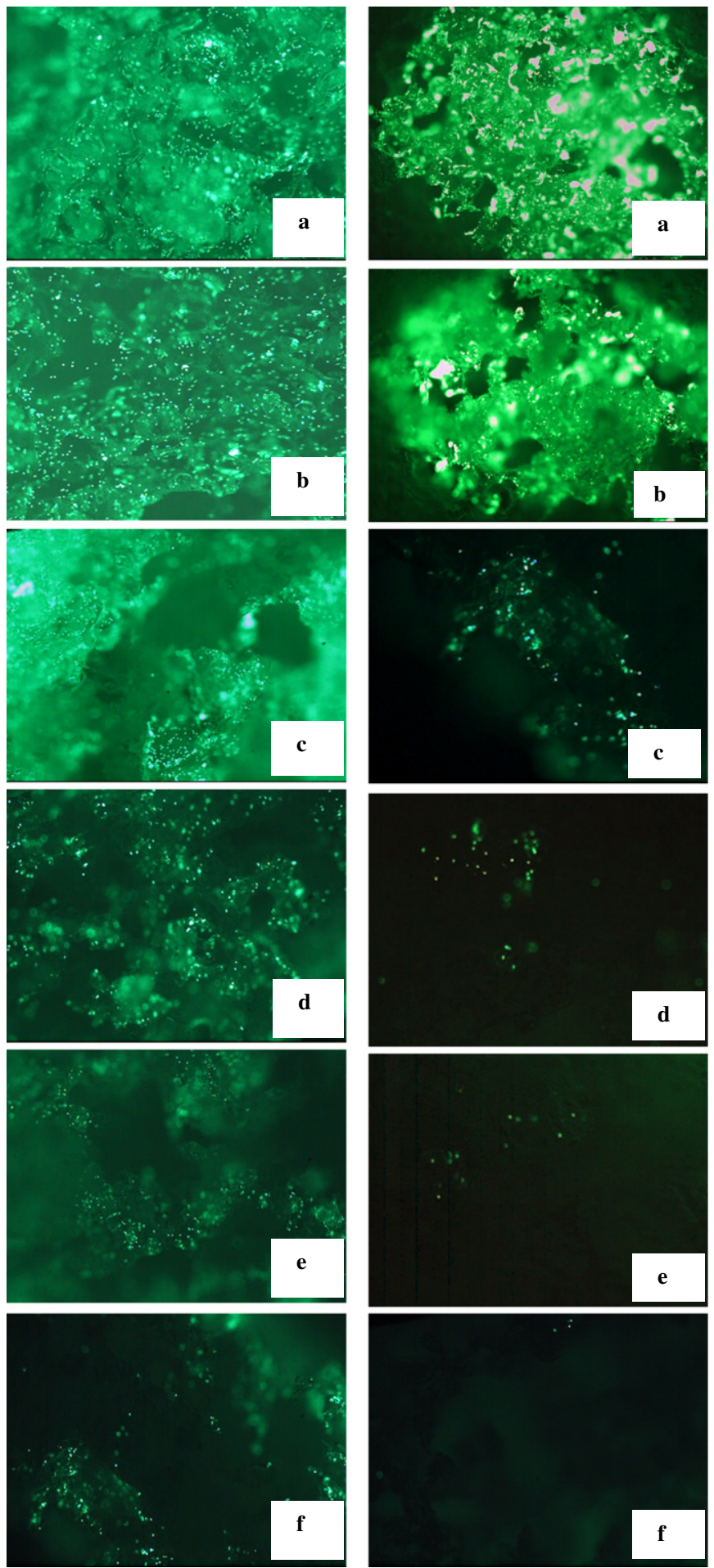

$100 \mu \mathrm{m}$ 


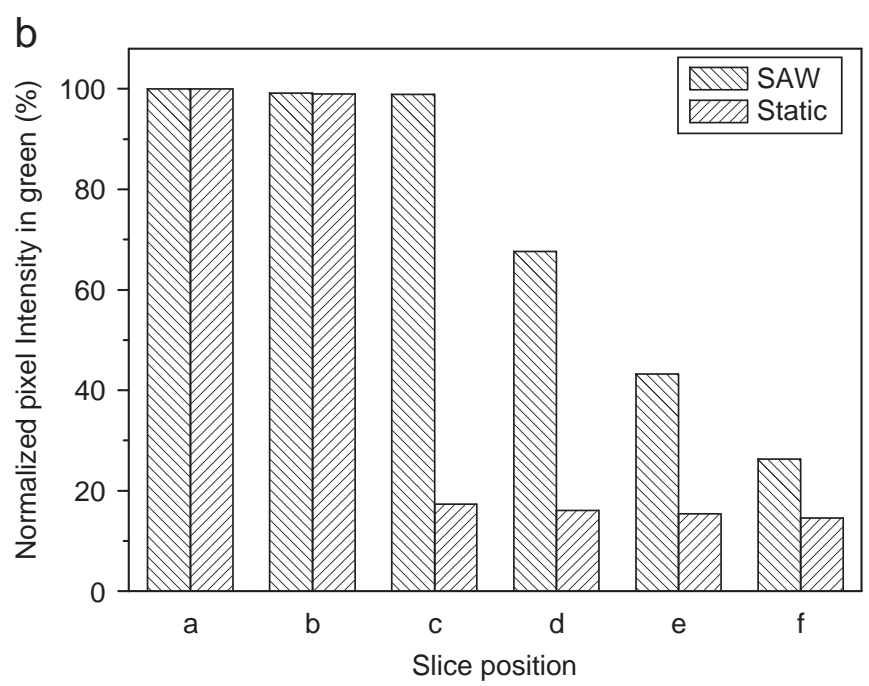

Fig. 5. (Continued)

in Fig. 2(b), it is possible to determine the spatial distribution of fluorescent particles within the scaffold and hence evaluate the uniformity of the cell seeding process. Cross-sectional images obtained from fluorescence microscopy of the successive slices of the scaffold are shown in Fig. 5. Images in the left column show the distribution of fluorescent particles in slices $(a-f)$ when the cell seeding process is driven by SAW actuation. The corresponding particle distribution in each slice, obtained via pixel intensity analysis of the fluorescent microscopic images in Fig. 5(a), is shown in Fig. 5(b). This is compared to the particle distribution in each slice using the static seeding method, shown in the right column of Fig. 5(a). In addition to rapid and efficient cell seeding, using SAW actuation to drive the particle suspension into the scaffold gives both greater particle penetration into the scaffold and a more uniform particle distribution. We observed in Fig. 5(a) that the particles are present in relative abundance in the last slice, i.e. slice (f), of the scaffold compared to same slice with the static seeding method. In the static method, most of the particles remained in the surface layer due to the inability of gravitational forces to overcome the large capillary pressure drop over the entire scaffold length. The penetration depth in this case was roughly $20 \%$ of the entire scaffold length; the particle's fluorescent intensity can be seen to drop drastically in slices (c-f).

\section{Conclusions}

We present a promising low-cost and miniaturizable alternative for driving a particle suspension into a porous cell scaffold through SAW actuation. The process is rapid; a particle suspension can be driven into a scaffold in about $10 \mathrm{~s}$, much quicker than that if the seeding occurred by pure diffusion alone or by other techniques proposed to date. In addition to rapid seeding, a higher seeding efficiency was also achieved in which higher particle concentrations were observed to perfuse deeper into the scaffold using SAW.
Moreover, the particle can be distributed in the scaffold more uniformly than when delivered by static method. The proposed SAW-driven method therefore holds promise for improving cell seeding for various applications in tissue and orthopaedic engineering.

\section{References}

[1] Soletti L, Nieponice A, Guan J, Stankus JJ, William WR, Vorp DA. A seeding device for tissue engineered tubular structures. Biomaterials 2006;27:4863-70.

[2] Wendt D, Marsano A, Jakob M, Heberer M, Martin I. Oscillating perfusion of cell suspensions through three-dimensional scaffolds enhances cell seeding efficiency and uniformity. Biotechnol Bioeng 2003;84:205-14.

[3] Li H, Chang J. Fabrication and characterization of bioactive wollastonite/PHBV composite scaffolds. Biomaterials 2004;25:5473-80.

[4] Li H, Chang J. Preparation and characterization of bioactive and biodegradable wollastonite/Poly(D,L-lactic acid) composite scaffolds. J Mater Sci: Mater Med 2004;15:1089-95.

[5] Maquet V, Martin D, Malgrange B, Franzen R, Schoenen J, Moonen $\mathrm{G}$, et al. Peripheral nerve regeneration using bioresorbable macroporous polylactide scaffolds. J Biomed Mater Res 2000;52: 639-51.

[6] Glicklis R, Shapiro L, Agbaria R, Merchuk JC, Cohen S. Hepatocyte behavior within three-dimensional porous alginate scaffolds. Biotechnol Bioeng 2000;67:344-53.

[7] Yoshikawa T, Ohgushi H, Tamai S. Immediate bone forming capability of prefabricated osteogenic hydroxyapatite. J Biomed Mater Res 1996;32:481-92.

[8] Dong J, Uemura T, Shirasaki Y, Tateishi T. Promotion of bone formation using highly pure porous b-TCP combined with bone marrow-derived osteoprogenitor cells. Biomaterials 2002;23:4493-502.

[9] Vunjak-Novakovic G, Obradovic B, Martin I, Bursac PM, Langer R, Freed LE. Dynamic cell seeding of polymer scaffolds for cartilage tissue engineering. Biotechnol Prog 1998;14:193-202.

[10] Zhao F, Ma T. Perfusion bioreactor system for human mesenchymal stem cell tissue engineering: dynamic cell seeding and construct development. Biotechnol Bioeng 2005;91:482-93.

[11] McFetridge PS, Daniel JW, Bodamyali T, Horrocks M, Chaudhuri JB. Preparation of porcine carotid arteries for vascular tissue engineering applications. J Biomed Mater Res A 2004;70:224-34.

[12] Kitagawa T, Yamaoka T, Iwase R, Murakami A. Three-dimensional cell seeding and growth in radial-flow perfusion bioreactor for in vitro tissue reconstruction. Biotechnol Bioeng 2005;93:947-57.

[13] Burg KJL, Holder Jr WD, Culberson CR, Beiler RJ, Greene KG, Loebsack AB, et al. Comparative study of seeding methods for threedimensional polymeric scaffolds. J Biomed Mater Res 2000;51:642-9.

[14] Kim BS, Putnam AJ, Kulik TJ, Mooney DJ. Optimizing seeding and culture methods to engineer smooth muscle tissue on biodegradable polymer matrices. Biotechnol Bioeng 1998;57:46-54.

[15] Sritharan K, Strobl CJ, Schneider MF, Wixforth A, Guttenberg Z. Acoustic mixing at low Reynolds numbers. Appl Phys Lett 2006;88:054102.

[16] Wixforth A, Strobl C, Gauer C, Toegl A, Scriba J, Guttenberg AV. Acoustic manipulation of small droplets. Anal Bioanal Chem 2004;379:982-91.

[17] Guttenberg Z, Rathgeber A, Keller S, Radler JO, Wixforth A, Kostur $\mathrm{M}$, et al. Flow profiling of a surface-acoustic-wave nanopump. P Phys Rev E Stat Nonlinear Soft Matter Phys 2004;70:056311.

[18] Li H, Yeo LY, Friend JR. Surface acoustic wave concentration of particle and bioparticle suspensions. Biomed Microdevice 2007; in press, doi:10.1007/s10544-007-9058-2.

[19] Morita T, Kurosawa MK, Higuchi T. Simulation of surface acoustic wave motor with spherical slider. IEEE Trans Ultrason Ferroelectr, Freq Control 1999;46:929-34. 
[20] Wixforth A. Acoustically driven planar microfluidics. Superlattice Microst 2004;33:389-96.

[21] Yang J, Shi GX, Bei JZ, Wang SG, Cao YL, Shang QX, et al. Fabrication and surface modification of macroporous poly(L-lactic acid) and poly(L-lactic-co-glycolic acid) (70/30) cell scaffolds for human skin fibroblast cell culture. J Biomed Mater Res 2002;62:438-46.

[22] Kurosawa M, Futami A, Higuchi T. Characteristics of liquids atomization using surface acoustic wave. In: Transducers' 97, Chicago: IEEE, 1997. p. 801-4.
[23] Hutmacher DW, Schantz T, Zein I, Ng KW, Teoh SH, Tan KC, Mechanical properties and cell cultural response of polycaprolactone scaffolds designed and fabricated via fused deposition modelling. J Biomed Mater Res 2001;55:203-16.

[24] Radel S, McLoughlin AJ, Gherardini L, Doblhoff-Dier O, Benes E. Viability of yeast cells in well controlled propagating and standing ultrasonic plane waves. Ultrasonics 2000;38:633-7.

[25] Cousins SM, Holownia P, Hawkes JJ, Limaye MS, Price CP, Keay PJ, et al. Plasma preparation from whole blood using ultrasound. Ultrasound Med Biol 2000;26:881-8. 